\title{
Simple model for the nonlinear radiation field of a free electron laser
}

\author{
Erik Hemsing $\odot^{*}$ \\ SLAC National Accelerator Laboratory, Menlo Park, California 94025, USA
}

(Received 25 September 2020; accepted 24 November 2020; published 4 December 2020)

It is shown that Jacobi elliptic function solutions of the nonlinear Duffing equation model the radiation field in a high-gain free electron laser through early saturation. After initial start-up, the field can be expressed equivalently with a hyperbolic secant. The model is derived for arbitrary detuning from resonance, which enables study of the spectral properties in the early nonlinear regime.

DOI: 10.1103/PhysRevAccelBeams.23.120703

\section{INTRODUCTION}

Linear free electron laser (FEL) theory has been extremely successful at describing the basic properties of the single pass, high-gain FEL in the start-up and exponential gain regimes. At saturation, however, nonlinear terms become dominant and the dynamics of the system is typically studied by numerical solutions to the underlying microscopic equations. Simplifications can yield insight into certain features of the saturation process, but the expressions must still generally be solved numerically [1-4]. Alternately, the system can be studied piecewise by dividing it into distinct dynamical regimes $[5,6]$.

Different approaches have been used to obtain unified analytic models for the FEL field in both the linear and nonlinear regimes, each targeting specific aspects. For example, the transition from exponential gain to saturation has been modeled by the Ginzburg-Landau equation [7,8] or with a logistic equation [9]. Werkhoven and Schep in [10] obtained somewhat involved but closed-form expressions for the resonant FEL field amplitude in terms of Jacobi elliptic functions that match well with numerical solutions deep into saturation. Solutions to the timedependent FEL equations in the limit of strong slippage have been obtained in the form of hyperbolic secant functions that evolve self-similarly $[11,12]$.

Here we show that the radiation field in a timeindependent high-gain FEL can be described from startup (either from electron beam bunching or from an external radiation field) through early saturation by the Jacobi elliptic function solutions to the unforced, undamped Duffing equation,

\footnotetext{
*ehemsing@slac.stanford.edu
}

Published by the American Physical Society under the terms of the Creative Commons Attribution 4.0 International license. Further distribution of this work must maintain attribution to the author(s) and the published article's title, journal citation, and DOI.

$$
A^{\prime \prime}-\alpha A+\beta A^{3}=0
$$

where $A$ is the scaled radiation field amplitude, and $\alpha, \beta>0$ are constants that depend on the detuning. This Duffing equation with its cubic nonlinearity describes the dynamical behavior of a myriad of Hamiltonian systems including nonlinear oscillators, so its use as a model for the nonlinear FEL may not be unexpected.

Starting from the standard 1D microscopic FEL equations, here we first derive a nonlinear equation for the complex field evolution. Using simple approximations, we show that the field can be modeled by (1) and find relatively simple Jacobi elliptic function solutions that closely match numerical solutions for arbitrary detuning. This provides an analytic description of the spectral evolution of the field as it saturates. With this model it is also straightforward to obtain the saturation power and saturation length. For small initial bunching $b_{0}$ the Jacobi elliptic functions can also be written in terms of hyperbolic secant functions in the exponential gain and nonlinear regimes. For example, at resonance, the field amplitude is well approximated by

$$
A(\bar{z})=\sqrt{\frac{3}{2}} \operatorname{sech}\left[\frac{\sqrt{3}}{2}\left(\bar{z}-\bar{z}_{s}\right)\right]
$$

where $\bar{z}_{s}=\frac{1}{\sqrt{3}} \ln \left(54 /\left|b_{0}\right|^{2}\right)$ is the saturation length. Finally, we show that the resonant solutions for the complex field also extend to the electron beam bunching factor and energy spread.

\section{NONLINEAR COLLECTIVE EQUATIONS}

We start with the microscopic 1D high-gain FEL equations, similar to those derived and used by Bonifacio et al., in Ref. [13] to obtain a collective nonlinear theory, 


$$
\begin{aligned}
& \frac{d \theta_{j}}{d \bar{z}}=\eta_{j} \\
& \frac{d \eta_{j}}{d \bar{z}}=a e^{i \theta_{j}}+a^{*} e^{-i \theta_{j}} \\
& \frac{d a}{d \bar{z}}=-\left\langle e^{-i \theta_{j}}\right\rangle+i \delta a,
\end{aligned}
$$

where $\theta$ and $\eta$ are the electron phase and scaled energy, $a$ is the scaled radiation field [14], $\bar{z}=2 k_{u} \rho z$ is the scaled distance along the FEL, $\rho$ is the FEL parameter [15], and $\delta=\left(\omega-\omega_{0}\right) / 2 \rho \omega_{0}$ is the frequency detuning. One can show that

$$
\begin{aligned}
\left\langle\eta_{j}\right\rangle+|a|^{2} & =C_{1} \\
\frac{\left\langle\eta_{j}^{2}\right\rangle}{2}+i\left(a\left\langle e^{i \theta_{j}}\right\rangle-a^{*}\left\langle e^{-i \theta_{j}}\right\rangle\right)-\delta|a|^{2} & =C_{2}
\end{aligned}
$$

are constants of motion, with $C_{1}$ and $C_{2}$ zero if the initial value of each of the terms is also zero [5].

The collective variable for the bunching factor is recognized as

$$
b=\left\langle e^{-i \theta_{j}}\right\rangle .
$$

Differentiating gives the standard collective energy variable $p$ :

$$
\frac{d b}{d \bar{z}}=-i\left\langle\frac{d \theta_{j}}{d \bar{z}} e^{-i \theta_{j}}\right\rangle=-i\left\langle\eta_{j} e^{-i \theta_{j}}\right\rangle=-i p
$$

The evolution of $p$ is also found by differentiating and using the definition for $d \eta_{j} / d \bar{z}$,

$$
\frac{d p}{d \bar{z}}=a-i\left\langle\eta_{j}^{2} e^{-i \theta_{j}}\right\rangle+a^{*}\left\langle e^{-2 i \theta_{j}}\right\rangle
$$

The second and third terms are ignored in the linear theory as they are both small prior to saturation, but are retained here to yield saturation effects.

The second term $\left\langle\eta_{j}^{2} e^{-i \theta_{j}}\right\rangle$ describes the first Fourier component of the energy spread of the beam about zero. We can use the fact that $\eta_{j}^{2}$ is only weakly correlated with $e^{-i \theta_{j}}$ up through early saturation such that the average of the product can be approximated as the product of the averages,

$$
\left\langle\eta_{j}^{2} e^{-i \theta_{j}}\right\rangle=\left\langle\eta_{j}^{2}\right\rangle\left\langle e^{-i \theta_{j}}\right\rangle=\sigma^{2} b .
$$

The real-valued collective variable $\sigma^{2}=\left\langle\eta_{j}^{2}\right\rangle$ gives the raw second moment of the beam energy distribution. The factorization ansatz in Eq. (8) here differs from that described in Ref. [13], where there the factorization is chosen to be in terms of the central second moment $\left\langle\left(\eta_{j}-\left\langle\eta_{j}\right\rangle\right)^{2} e^{-i \theta_{j}}\right\rangle=\left\langle\left(\eta_{j}-\left\langle\eta_{j}\right\rangle\right)^{2}\right\rangle\left\langle e^{-i \theta_{j}}\right\rangle$. The latter choice adds highly nonlinear terms and more complicated expressions [e.g., Eq. (3) in Ref. [7]]. The difference between ansätze appears negligible in the early saturation regime as both closely match exact numerical solutions to Eq. (3). See Fig. 1 here and in Ref. [13].

The third term in (7) includes the bunching at the second harmonic $\left\langle e^{-2 i \theta_{j}}\right\rangle$. Its contribution to the nonlinear equations by way of the collective variables is included by assuming that

$$
\left\langle e^{-2 i \theta_{j}}\right\rangle \approx\left\langle e^{-i \theta_{j}}\right\rangle\left\langle e^{-i \theta_{j}}\right\rangle=b^{2}
$$

The validity of this approximation is also shown in Fig. 1. The inclusion of this term helps to better model the nonlinear behavior near saturation, including specific features of the saturated FEL gain spectrum. Overall, results from numerical particle simulations of the governing equations (3) show that the approximations in (8) and (9) are reasonable in early saturation, thereby enabling a concise mathematical framework in terms of collective variables that leads to the simple analytic model for the FEL up through the early nonlinear regime.

Using (4) for a cold beam $\left[\sigma^{2}(0)=0\right]$ and an FEL that grows from initial bunching rather than an external seed $\left[a(0)=a_{0}=0\right]$ we have $C_{2}=0$, and $\sigma^{2}=\left\langle\eta_{j}^{2}\right\rangle$ becomes

$$
\sigma^{2}(\bar{z})=2 i\left(a^{*} b-a b^{*}\right)+2 \delta|a|^{2} .
$$
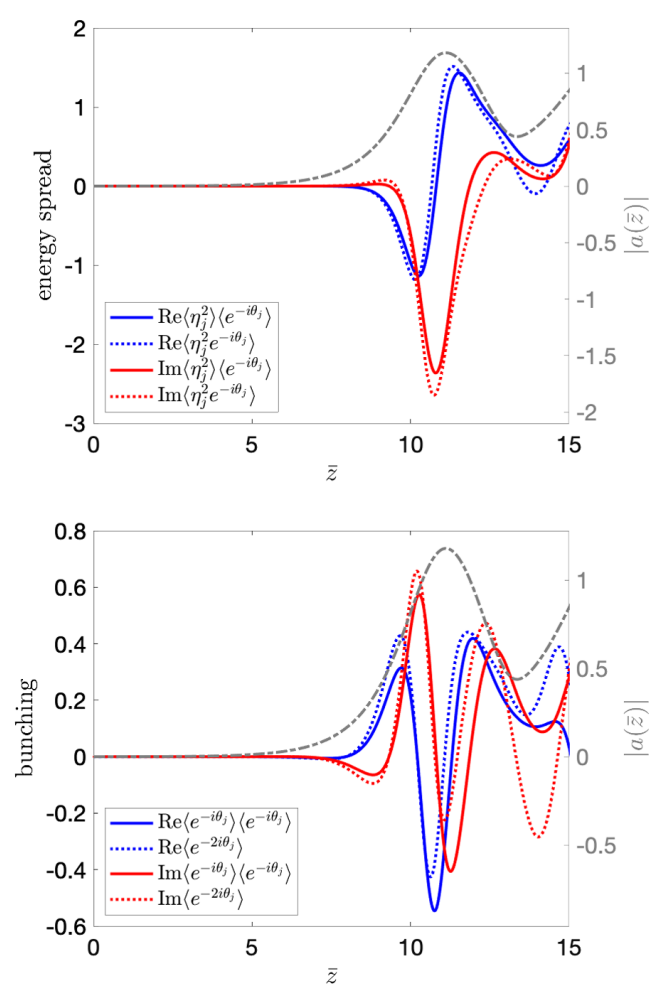

FIG. 1. Check of factorization ansätze in Eqs. (8) and (9). Results are from the numerical solution to the microscopic equations in (3) with initial condition $\left|b_{0}\right|=5 \times 10^{-4}$. 
Combining terms yields a set of three equations of collective variables that models the high-gain FEL from start-up through exponential gain and into early saturation,

$$
\begin{aligned}
& \frac{d b}{d \bar{z}}=-i p \\
& \frac{d a}{d \bar{z}}=-b+i \delta a \\
& \frac{d p}{d \bar{z}}=a+2 b\left[\left(a^{*} b-a b^{*}\right)-i \delta|a|^{2}\right]+a^{*} b^{2} .
\end{aligned}
$$

This can be condensed into a single equation for the field,

$$
\begin{aligned}
& i \frac{d^{3} a}{d \bar{z}^{3}}+a+\delta \frac{d^{2} a}{d \bar{z}^{2}} \\
& \quad=\left(\frac{d a}{d \bar{z}}-i \delta a\right)\left[2 a \frac{d a^{*}}{d \bar{z}}-3 a^{*} \frac{d a}{d \bar{z}}+3 i \delta|a|^{2}\right] .
\end{aligned}
$$

From this equation we will derive approximate solutions for $a(\bar{z})$ in terms of Jacobi elliptic functions.

\section{LINEAR REGIME}

Solutions for the field in the linear regime will be helpful in obtaining solutions that extend into the nonlinear regime. In the linear regime the energy spread and harmonic terms that comprise the right-hand side of (12) are negligible, so the field evolution is

$$
i a^{\prime \prime \prime}+a+\delta a^{\prime \prime}=0 .
$$

With $a_{0}, p_{0}=0$, the general solutions are [16]

$$
a(\bar{z})=-i b_{0} \sum_{l=1}^{3} \frac{e^{-i \mu_{l} \bar{z}}}{3 \mu_{l}+2 \delta},
$$

where the initial bunching $b_{0}$ can be complex valued and where each $\mu_{l}$ is a solution to the cubic dispersion equation

$$
\mu^{3}-1+\delta \mu^{2}=0
$$

that satisfies $\sum_{1}^{3} \mu_{l}=-\delta$ and $\prod_{1}^{3} \mu_{l}=1$. The exponentially growing mode solution is

$$
\mu=\xi \frac{-1+i \sqrt{3}}{2}-\frac{\delta}{3}-\frac{\delta^{2}}{9 \xi} \frac{1+i \sqrt{3}}{2},
$$

where $\xi=\left(27-2 \delta^{3}+3 \sqrt{81-12 \delta^{3}}\right)^{1 / 3} / 54^{1 / 3}$, and $\xi=1$ at $\delta=0$. Exponential growth occurs only for detuning less than a critical value $\delta<\delta_{c}=3 / 4^{1 / 3}$ [17]. In this domain the real and imaginary components $\mu=\mu_{R}+i \mu_{I}$ are related by

$$
\mu_{I}^{2}=\frac{\mu_{R}^{3}+\delta \mu_{R}^{2}-1}{3 \mu_{R}+\delta}=\mu_{R}\left(3 \mu_{R}+2 \delta\right) .
$$

Expanding (16) to second order in $\delta$ gives $\mu \approx$ $-\frac{1}{2}\left(1+\frac{\delta}{3}\right)^{2}+i \frac{\sqrt{3}}{2}\left(1-\frac{\delta^{2}}{9}\right)$, which shows that the field intensity of the growing mode has an approximately Gaussian detuning spectrum,

$$
|a|^{2} \propto\left|e^{-i \mu \bar{z}}\right|^{2} \approx e^{\sqrt{3} \bar{z}-\delta^{2} / 2 \sigma_{\delta}^{2}}
$$

with rms bandwidth

$$
\sigma_{\delta}^{2}=\frac{3 \sqrt{3}}{2 \bar{z}} .
$$

\section{NONLINEAR SOLUTIONS: THE DUFFING EQUATION}

Returning to the full nonlinear equation for the field evolution in (12), closed form approximate expressions are obtained by assuming that the complex field can be written in terms of an amplitude and phase, both real valued:

$$
a(\bar{z})=A(\bar{z}) e^{i \phi(\bar{z})} .
$$

In the exponential growth regime (i.e., after start-up), Eqs. (14) and (16) show that the field phase is linear with $\bar{z}$, and has a slope $-\mu_{R}$ that depends on the detuning. Inspired by this dependence we model the phase as

$$
\phi(\bar{z})=-\tilde{\mu}_{R} \bar{z}+\phi_{0},
$$

where $\phi_{0}$ is determined by the initial conditions to keep $A$ real valued, and

$$
-\tilde{\mu}_{R}=\frac{\xi}{2}+\frac{\delta}{3}+\frac{\delta^{2}}{18 \xi} .
$$

This is plotted in Fig. 2. Note that $\tilde{\mu}_{R}=\mu_{R}$ for $\delta<\delta_{c}$ but, unlike $\mu_{R}$, is smooth at $\delta=\delta_{c}$.

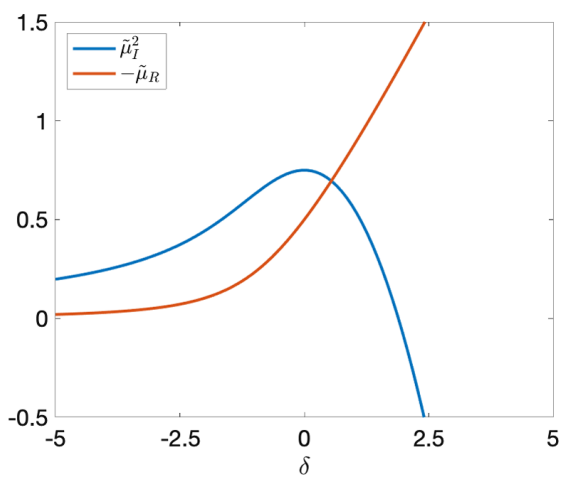

FIG. 2. Continuous differential gain parameter $\tilde{\mu}_{I}^{2}$ from (24) and phase slope $-\tilde{\mu}_{R}$ from (22). 
Inserting $a(\bar{z})$ from (20) into the nonlinear equation for the field evolution (12) and setting the imaginary term to zero reveals our unforced, undamped Duffing equation from (1) that models the amplitude evolution through early saturation,

$$
A^{\prime \prime}-\alpha A+\beta A^{3}=0,
$$

where the coefficients are

$$
\alpha=\tilde{\mu}_{R}\left(3 \tilde{\mu}_{R}+2 \delta\right)=\tilde{\mu}_{I}^{2}, \quad \beta=-2 \tilde{\mu}_{R}-\frac{4 \delta}{3} .
$$

Using the relation between $\mu_{R}$ and $\mu_{I}$ in (17) we define $\tilde{\mu}_{I}$ in (24) in terms of $\tilde{\mu}_{R}$, where $\tilde{\mu}_{I}=\mu_{I}$ identically for $\delta<\delta_{c}$ but, like $\tilde{\mu}_{R}$, it is also smooth across $\delta=\delta_{c}$ (see Fig. 2). The coefficient $\alpha=\tilde{\mu}_{I}^{2}$ of the linear term in (23) is thus related directly to the term responsible for gain in the linear FEL theory. This is to be expected, since if the nonlinear term $A^{3}$ is small, solutions to (23) are $A(\bar{z}) \propto e^{\tilde{\mu}_{1} \bar{z}}$, as required in the exponential growth regime. The nonlinearity, set by $\beta$, produces the saturation effect once the field strength becomes large enough.

Equation (23) is a well-known nonlinear differential equation with analytic solutions described by the Jacobi elliptic functions [18]. General solutions for arbitrary $\alpha, \beta>0$ are given by

$$
A(\bar{z})=Y \mathbf{c n}(\kappa \bar{z}+\psi \mid n),
$$

where $\mathbf{c n}$ is the cosinelike Jacobi elliptic function. In the convention of [19], $\kappa$ is called the frequency and $n$ the parameter (the so-called modulus is $\sqrt{n}$ ). They are related by

$$
\kappa^{2}=\beta Y^{2}-\alpha, \quad n=\frac{\beta Y^{2}}{2 \kappa^{2}} .
$$

The peak amplitude is given by

$$
Y^{2}=\frac{\alpha \pm \sqrt{\left(\alpha-\beta A_{0}^{2}\right)^{2}+2 \beta\left(A_{0}^{\prime}\right)^{2}}}{\beta} .
$$

The phase angle $\psi$ satisfies a general relation subject to initial conditions,

$$
\frac{\operatorname{sn}(\psi \mid n) \mathbf{d n}(\psi \mid n)}{\mathbf{c n}(\psi \mid n)}=-\frac{A_{0}^{\prime}}{A_{0} \kappa} .
$$

In the $a_{0}=0$ case considered here, $A(0)=A_{0}=0$ and $\psi=-K(n)$, where $K(n)$ is the complete elliptic integral of the first kind. The sign is chosen in anticipation of matching the linear FEL solutions in tandem with the proper choice of $A_{0}^{\prime}$, which is proportional to $\left|b_{0}\right|$ and small. Taking the upper sign in (27) [20], $Y$ and $\kappa$ are independent of $A_{0}^{\prime}$ to lowest order,
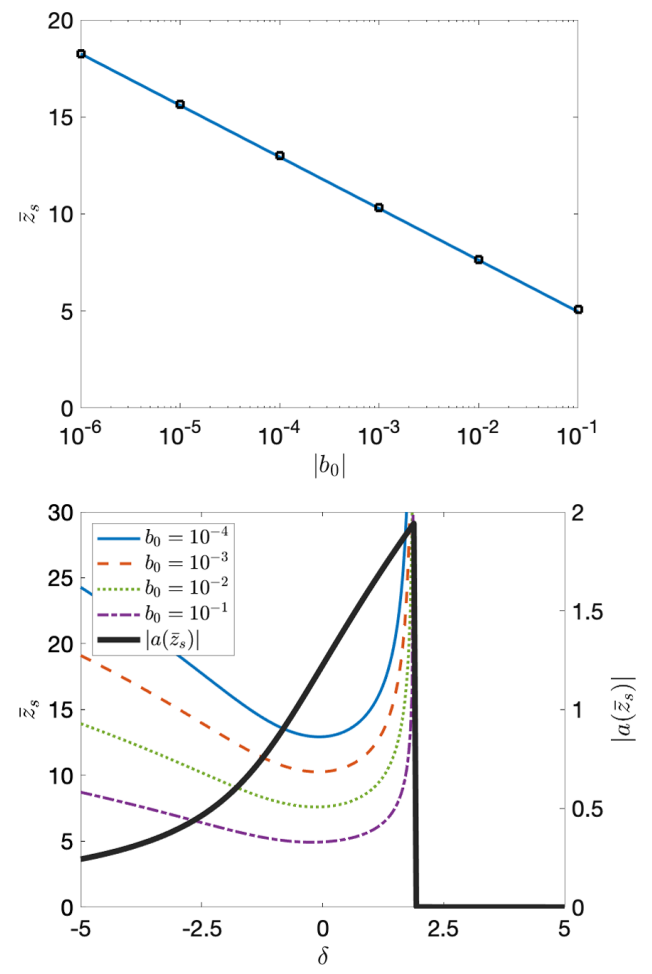

FIG. 3. Top: saturation length at $\delta=0$ as a function of initial bunching amplitude from (38) (blue line) and from solutions to the microscopic equations in (3) (squares). Bottom: saturation length $\bar{z}_{s}$ from (34) for different values of initial bunching. The saturated field amplitude $\left|a\left(\bar{z}_{s}\right)\right|$ is also shown.

$$
Y \approx \sqrt{\frac{2 \alpha}{\beta}}=\sqrt{-3 \tilde{\mu}_{R}}, \quad \kappa \approx \sqrt{\alpha}=\tilde{\mu}_{I} .
$$

The peak amplitude $Y$ is related to the phase slope from (22), while the frequency $\kappa$ relates to the differential gain in (24). For $n$, the next order term is needed to properly account for the initial conditions,

$$
n \approx 1-\frac{\left(A_{0}^{\prime}\right)^{2} \beta}{2 \alpha^{2}}=1+\frac{\left(A_{0}^{\prime}\right)^{2}}{3 \tilde{\mu}_{R} \tilde{\mu}_{I}^{2}}
$$

The parameter $n$ is generally close to unity, enabling two simplifying approximations. The first is

$$
K(n)=\ln \left(\frac{4}{\sqrt{1-n}}\right)
$$

The second is that $\operatorname{cn}(x \mid n) \approx \operatorname{sech}(x)$ in the exponential growth and early saturation regimes. The sech solution in the exponential regime is then $a(\bar{z})=\frac{A_{0}^{\prime}}{2 \tilde{\mu}_{I}} e^{\kappa \bar{z}-i \tilde{\mu}_{R} \bar{z}+i \phi_{0}}$ while from (14) the field should be $a(\bar{z})=-i \frac{b_{0}}{3 \mu+2 \delta} e^{-i \mu \bar{z}}$. Equating these expressions yields the initial condition required to match the exponential growth from linear theory, 


$$
A_{0}^{\prime}=-2 i \frac{b_{0} \tilde{\mu}_{I}}{3 \mu+2 \delta} e^{-i \phi_{0}}=\zeta e^{-i \phi_{0}}
$$

where the phase offset is

$$
\phi_{0}=-i \ln (\zeta /|\zeta|)
$$

With the cn Jacobi functions it is straightforward to calculate the saturation point because they are $4 K(n)$ periodic. Namely, the saturation point of $\mathbf{c n}(\kappa \bar{z}-K(n) \mid n)$ is given by $\mathbf{c n}(0 \mid n)=1$, or, with (31) and (32), at the location

$$
\bar{z}_{s}=\frac{K(n)}{\kappa}=\frac{1}{2 \tilde{\mu}_{I}} \ln \left(-\frac{12 \tilde{\mu}_{R}|3 \mu+2 \delta|^{2}}{\left|b_{0}\right|^{2}}\right) .
$$

The saturated field amplitude is then simply $\left|a\left(\bar{z}_{s}\right)\right|=Y$, which also depends on the detuning and is shown in Fig. 3. We note the similarity in the shape and amplitude of $\left|a\left(\bar{z}_{s}\right)\right|$ with the equilibrium saturation field calculated from a statistical theory in Ref. [21]. The saturation point $\bar{z}_{s}$ scales logarithmically with the initial bunching, and depends on the detuning though $\mu, \tilde{\mu}_{R}$, and $\tilde{\mu}_{I}$. Figure 3 also shows the saturation length for different $b_{0}$ as a function of $\delta$. Positive detuning increases the output power despite the longer associated gain lengths.

Combining everything together yields an expression for the complex field,

$$
\begin{aligned}
a(\bar{z}) & =\sqrt{-3 \tilde{\mu}_{R}} \mathbf{c n}\left[\tilde{\mu}_{I}\left(\bar{z}-\bar{z}_{s}\right) \mid 1-16 e^{-2 \tilde{\mu}_{I} \bar{z}_{s}}\right] e^{-i \tilde{\mu}_{R} \bar{z}+i \phi_{0}} \\
& =\frac{2 b_{0}}{3 \mu+2 \delta} \mathbf{c n}\left(i \tilde{\mu}_{I} \bar{z}+\frac{\pi}{2} \mid 16 e^{-2 \tilde{\mu}_{I} \bar{z}_{s}}\right) e^{-i \tilde{\mu}_{R} \bar{z}}
\end{aligned}
$$

The second, more compact expression has been obtained with the Jacobi elliptic function transformations. This is the main result of this paper. It describes the complex field of the FEL as a function of $\bar{z}$ and $\delta$ through early saturation due to initial bunching.

The detuning spectrum for $|a(\bar{z})|$ at different $\bar{z}$ positions is shown in Fig. 4 as calculated from microscopic particle equations in (3), the nonlinear field equation in (12), and Jacobi functions in (35). There is good agreement between all three up through early saturation. In the linear regime the spectrum resembles the Gaussian approximation from Eq. (18). As the system saturates, the spectrum begins to fragment, with the development of a narrowed spectral region for $\delta>0$ that supports increased output power [22].

Figure 5 shows the normalized detuning spectrum for a fixed initial bunching amplitude. The Jacobi function solutions in (35) reproduce the essential features of the spectrum, including the $1 / \sqrt{\bar{z}}$ bandwidth narrowing in the linear regime and the bifurcation at saturation. The evolution of the relative bandwidth can be seen clearly in Fig. 6, where again there is good agreement. The increase in bandwidth at saturation is well known and accompanies a reduction in energy fluctuations in radiation pulses starting from noise, which scale like $1 / \sqrt{\sigma_{\delta}}$ in the linear regime [23]. (a)

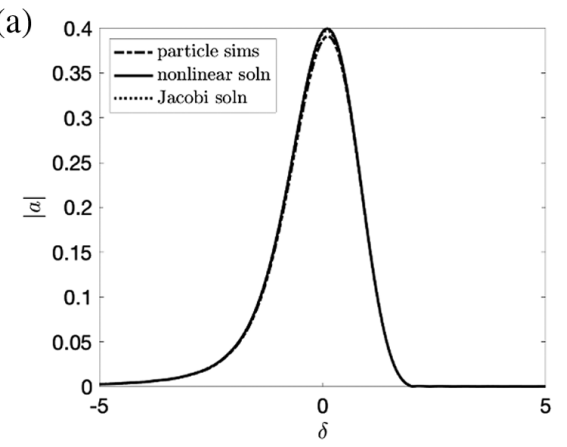

(d)

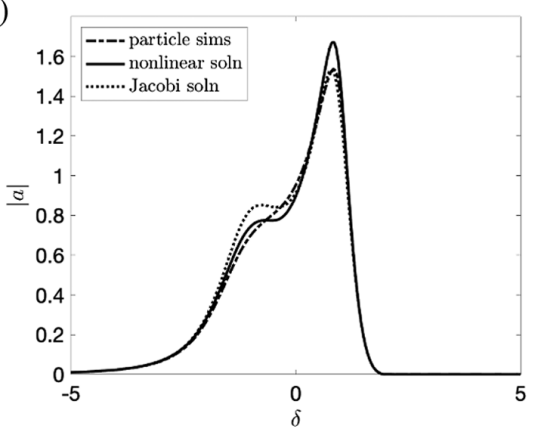

(b)

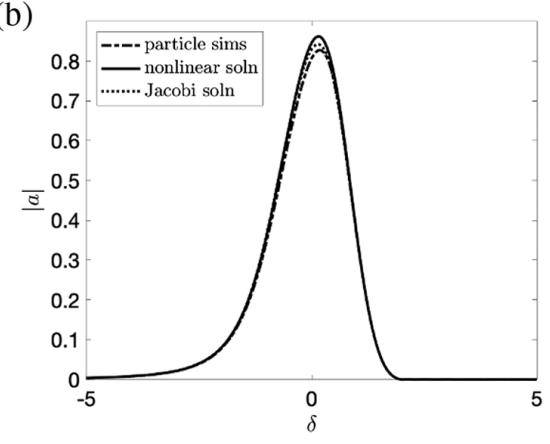

(e)

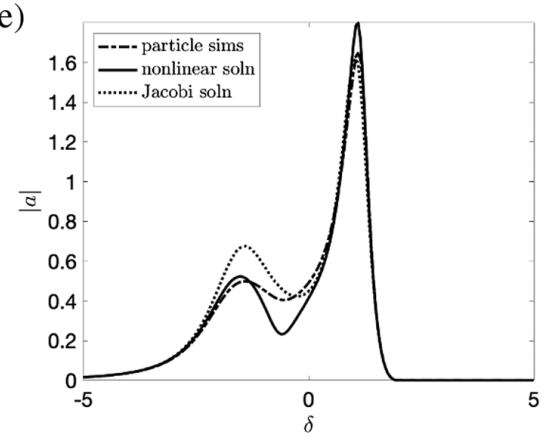

(c) 1.0

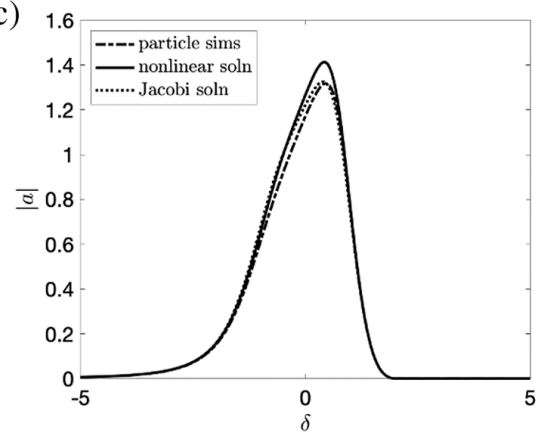

(f)

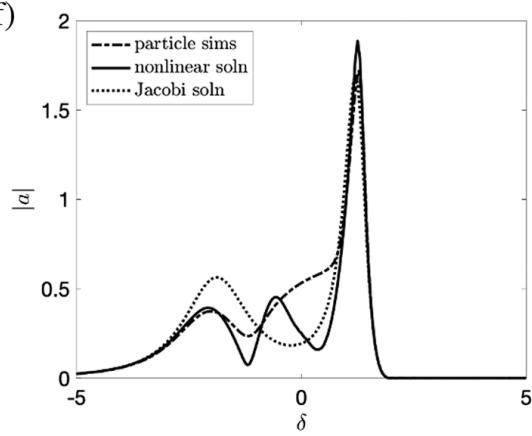

FIG. 4. Detuning spectra for (a) $\bar{z}=9$, (b) 10 , (c) 11 , (d) 12 , (e) 13 , and (f) 14 . Here $\left|b_{0}\right|=5 \times 10^{-4}$ with $\rho=10^{-3}$, and saturation occurs at $\bar{z}_{s}=11$. The good agreement between solutions begins to dissolve a couple of gain lengths $(\Delta \bar{z} \approx 2)$ after field saturation, defined for $\delta=0$. 

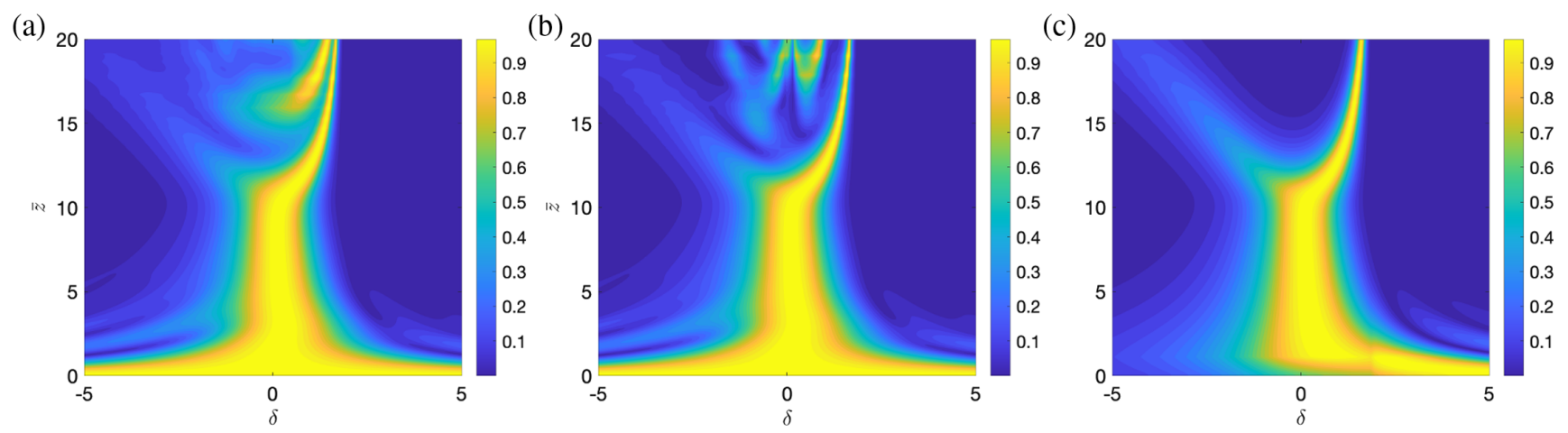

FIG. 5. Normalized detuning spectra from (a) microscopic particle simulations, (b) numerical solutions to the nonlinear collective equations, and (c) Jacobi elliptic functions. Here $\left|b_{0}\right|=5 \times 10^{-4}$, which gives saturation at $\bar{z}_{s}=11$.

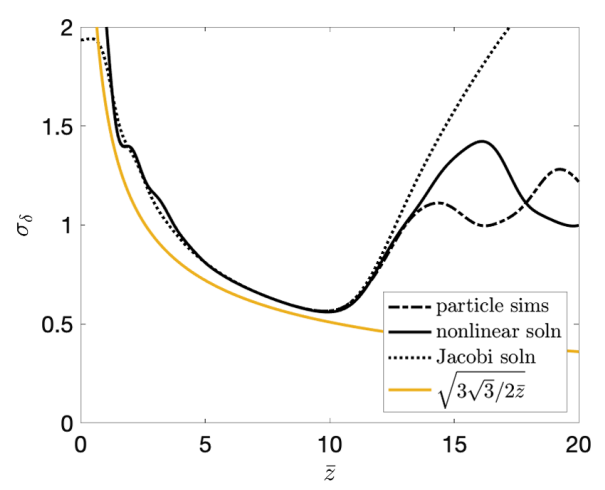

FIG. 6. Evolution of the relative rms detuning bandwidth with $\left|b_{0}\right|=5 \times 10^{-4}$ for different solutions, including the Gaussian approximation for the linear regime in Eq. (19).

After lethargy, the field in (35) can also be written as

$$
a(\bar{z})=\sqrt{-3 \tilde{\mu}_{R}} \operatorname{sech}\left[\tilde{\mu}_{I}\left(\bar{z}-\bar{z}_{s}\right)\right] e^{-i \tilde{\mu}_{R} \bar{z}+i \phi_{0}}
$$

for $\left|b_{0}\right| \ll 1$. At resonance, from this one can obtain Eq. (2). This solution bears resemblance to the sech solutions derived for the time-dependent FEL in the short pulse limit in Refs. [11,12].

\section{NONLINEAR SOLUTIONS FOR $\delta=0$}

At zero detuning there are significant simplifications. The gain parameter in (16) is

$$
\mu=-\frac{1}{2}+i \frac{\sqrt{3}}{2}=\tilde{\mu}_{R}+i \tilde{\mu}_{I}
$$

such that $\alpha=3 / 4, \beta=1$, and $Y=\sqrt{3 / 2}$. The Duffing equation becomes $A^{\prime \prime}=\frac{3}{4} A-A^{3}$. The equilibrium solution for the power is $A^{2}=3 / 4$, which is close to the value given in Ref. [5].
The saturation point for the resonant field from (34) is

$$
\bar{z}_{s}=\frac{1}{\sqrt{3}} \ln \left(\frac{54}{\left|b_{0}\right|^{2}}\right) .
$$

The dependence of $\bar{z}_{s}$ at resonance on the initial bunching is shown in Fig. 3. The complex field from (35) is therefore

$$
a(\bar{z})=\frac{2 b_{0}}{3 \mu} \mathbf{c n}\left(i \frac{\sqrt{3}}{2} \bar{z}+\left.\frac{\pi}{2}\left|\frac{8}{27}\right| b_{0}\right|^{2}\right) e^{i \bar{z} / 2} .
$$

With solutions for the resonant field it is straightforward to also write the other collective variables in terms of the Jacobi elliptic functions. Using $b=-d a / d \bar{z}$ from (11) and the derivative property of elliptic functions $\frac{d \mathbf{c n}(u \mid m)}{d u}=$ $-\mathbf{s n}(u \mid m) \mathbf{d n}(u \mid m)$, the bunching factor is

$$
\begin{aligned}
b(\bar{z})= & -\frac{i b_{0}}{3 \mu} e^{i \bar{z} / 2}\left[\operatorname{cn}\left(i \frac{\sqrt{3}}{2} \bar{z}+\left.\frac{\pi}{2}\left|\frac{8}{27}\right| b_{0}\right|^{2}\right)\right. \\
& -\sqrt{3} \operatorname{sn}\left(i \frac{\sqrt{3}}{2} \bar{z}+\left.\frac{\pi}{2}\left|\frac{8}{27}\right| b_{0}\right|^{2}\right) \\
& \left.\times \operatorname{dn}\left(i \frac{\sqrt{3}}{2} \bar{z}+\left.\frac{\pi}{2}\left|\frac{8}{27}\right| b_{0}\right|^{2}\right)\right] .
\end{aligned}
$$

Using the field approximation in (20), the energy spread parameter is simply

$$
\sigma^{2}(\bar{z})=2|a(\bar{z})|^{2}
$$

using (39). The evolution of $|a(\bar{z})|,|b(\bar{z})|$, and $\sigma(\bar{z})$ at resonance is shown in Fig. 7. Good agreement through early saturation is found with both the microscopic equations (3) and the nonlinear collective equations in (11). A couple of gain lengths beyond field saturation the solutions diverge, particularly the microscopic equations. Both the collective equation solutions and Jacobi functions fail to properly reproduce the postsaturation oscillations produced by synchrotron motion. 
(a)

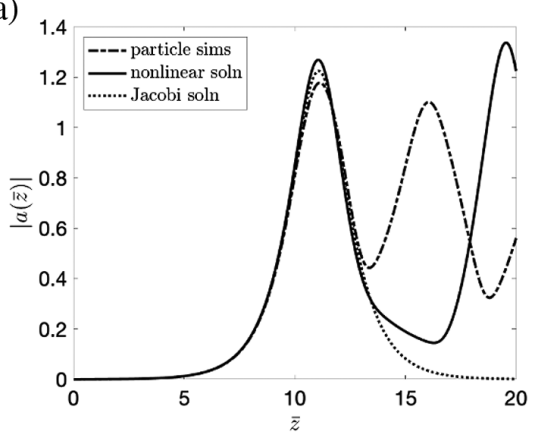

(d)

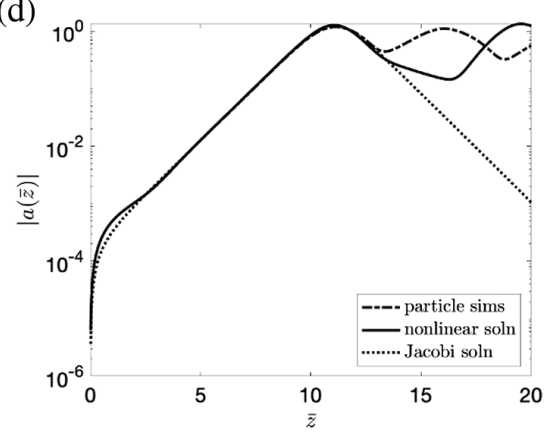

(b)

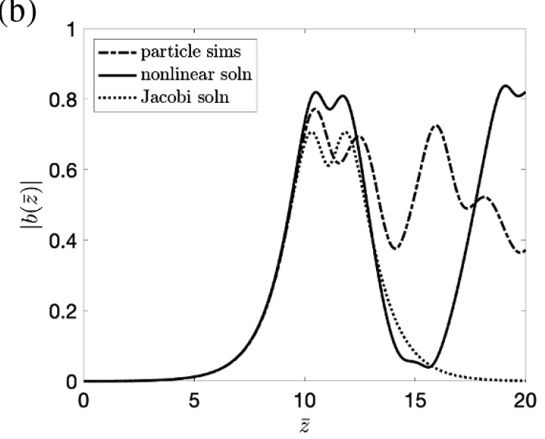

(e)

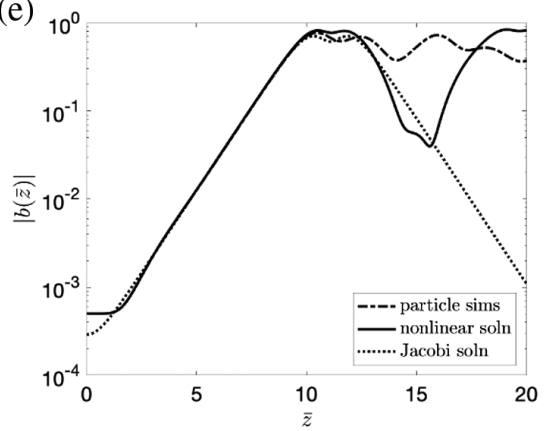

(c)

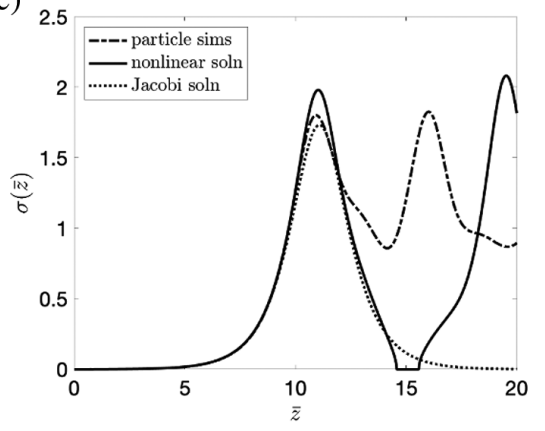

(f)

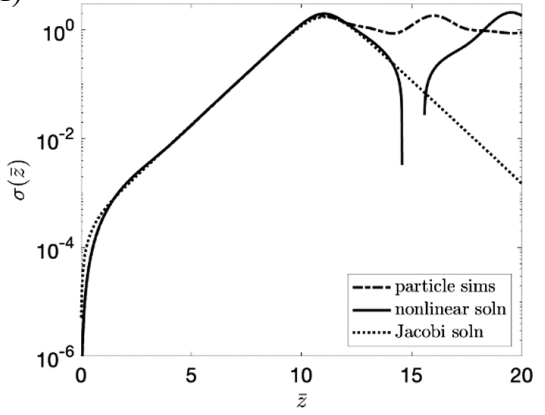

FIG. 7. Evolution of the FEL field amplitude (left), bunching (middle), and energy spread (right) calculated from microscopic particle simulations of Eq. (3) (dash-dotted line), numerical solutions to the nonlinear collective variable equations in (11) (solid line), and the analytic Jacobi elliptic function solutions in (39)-(41) (dotted line). Here $\delta=0,\left|b_{0}\right|=5 \times 10^{-4}, \bar{z}_{s}=11$, and $\rho=10^{-3}$. The bottom row shows $\log$ plots of the top row.

With (38), it is straightforward to obtain the value of the field, bunching, and energy spread at field saturation,

$\left|a\left(\bar{z}_{s}\right)\right|=\sqrt{\frac{3}{2}}, \quad\left|b\left(\bar{z}_{s}\right)\right|=\frac{1}{2} \sqrt{\frac{3}{2}}, \quad \sigma\left(\bar{z}_{s}\right)=\sqrt{3}$.

A couple of points are worth mention. First, inspection of the collective bunching variable in (40) gives an initial value $b(0)=i b_{0} / \sqrt{3} \mu$, such that there is slight disagreement between the analytic and numerical solutions in the early values of $a, b$, and $\sigma$ in the lethargy regime, as seen in Fig. 7. This is due to our choice of initial condition $A_{0}^{\prime}$ for the Duffing equation, which was made in order that the Jacobi solutions better match the full solutions in the linear and saturation regimes. Second, while (40) and (41) are reasonable models for $b$ and $\sigma$ at resonance, unlike $a$, the description does not accurately generalize $b$ and $\sigma$ for arbitrary detuning.

\section{SOLUTIONS FOR $b_{0}=0, a_{0} \neq 0$}

It is straightforward to apply the same procedure and obtain solutions to the Duffing equation for an FEL amplifier where the initial bunching can be neglected and the input field is $a_{0}$. In this case, $\alpha=\tilde{\mu}_{R}\left(3 \tilde{\mu}_{R}+2 \delta\right)+2 \delta\left|a_{0}\right|^{2}$ and $Y=A_{0}=\frac{2 a_{0} \mu}{3 \mu+2 \delta} e^{-i \phi_{0}}$. For $\left|a_{0}\right| \ll 1$ the field is then $a(\bar{z})=\frac{2 a_{0} \mu}{3 \mu+2 \delta} \mathbf{c n}\left(\left.i \tilde{\mu}_{I} \bar{z}\left|\frac{4}{3 \tilde{\mu}_{R}}\right| \frac{a_{0} \mu}{3 \mu+2 \delta}\right|^{2}\right) e^{-i \tilde{\mu}_{R} \bar{z}}$,

and saturation occurs at

$$
\bar{z}_{s}=\frac{1}{2 \tilde{\mu}_{I}} \ln \left(-\frac{12 \tilde{\mu}_{R}|3 \mu+2 \delta|^{2}}{\left|a_{0} \mu\right|^{2}}\right) .
$$

\section{CONCLUSIONS}

We have shown that the complex radiation field in a 1D high-gain FEL can be described by a Jacobi elliptic function or hyperbolic secant through the early nonlinear regime. The model is derived for arbitrary detuning, which enables study of the spectral properties of the system as it saturates. At resonance, the description extends to the bunching factor and beam energy spread, and provides an accurate estimate of the saturation length and saturation power. The presented approach may provide insight into a more general description that extends into deep saturation and is time dependent.

\section{ACKNOWLEDGMENTS}

The author thanks W. M. Fawley, C. Pellegrini, A. Halavanau, Z. Huang, B. W. J. McNeil, N. Sudar, and Z. Zhang for helpful conversations. This work was supported 
by U.S. Department of Energy Contract No. DE-AC0276SF00515 and Award No. 2017-SLAC-100382.

[1] Z. Huang and K.-J. Kim, Transverse and temporal characteristics of a high-gain free-electron laser in the saturation regime, Nucl. Instrum. Methods Phys. Res., Sect. A 483, 504 (2002).

[2] S. Krinsky, Saturation of a high-gain single-pass FEL, Nucl. Instrum. Methods Phys. Res., Sect. A 528, 52 (2004).

[3] N. Vinokurov, Z. Huang, O. Shevchenko, and K.-J. Kim, Quasilinear theory of high-gain FEL saturation, Nucl. Instrum. Methods Phys. Res., Sect. A 475, 74 (2001).

[4] C. Maroli, N. Sterpi, M. Vasconi, and R. Bonifacio, Threemode treatment of a high-gain steady-state free-electron laser, Phys. Rev. A 44, 5206 (1991).

[5] R. L. Gluckstern, S. Krinsky, and H. Okamoto, Analysis of the saturation of a high-gain free-electron laser, Phys. Rev. E 47, 4412 (1993).

[6] G. Dattoli, P. L. Ottaviani, and S. Pagnutti, High gain amplifiers: Power oscillations and harmonic generation, J. Appl. Phys. 102, 033103 (2007).

[7] R. Bonifacio, C. Maroli, and A. Dragan, A landauginzburg equation for a high-gain free electron laser, Opt. Commun. 76, 353 (1990).

[8] S. Y. Cai and A. Bhattacharjee, Ginzburg-Landau equation: A nonlinear model for the radiation field of a free-electron laser, Phys. Rev. A 43, 6934 (1991).

[9] G. Dattoli, L. Giannessi, P. L. Ottaviani, and C. Ronsivalle, Semi-analytical model of self-amplified spontaneous-emission free-electron lasers, including diffraction and pulsepropagation effects, J. Appl. Phys. 95, 3206 (2004).

[10] G. van Werkhoven and T. Schep, Non-linear macroscopic equations for pulse evolution in a high-gain Compton FEL, Nucl. Instrum. Methods Phys. Res., Sect. A 358, 485 (1995).

[11] R. Bonifacio, L. De Salvo Souza, P. Pierini, and N. Piovella, The superradiant regime of a FEL: Analytical and numerical results, Nucl. Instrum. Methods Phys. Res., Sect. A 296, 358 (1990).

[12] N. Piovella, A hyperbolic secant solution for the superradiance in free electron lasers, Opt. Commun. 83, 92 (1991).

[13] R. Bonifacio, F. Casagrande, and L. De Salvo Souza, Collective variable description of a free-electron laser, Phys. Rev. A 33, 2836 (1986).

[14] K.-J. Kim, Z. Huang, and R. Lindberg, Synchrotron Radiation and Free-Electron Lasers: Principles of Coherent X-Ray Generation (Cambridge University Press, Cambridge, England, 2017).

[15] R. Bonifacio, C. Pellegrini, and L. Narducci, Collective instabilities and high-gain regime in a free electron laser, Opt. Commun. 50, 373 (1984).

[16] R. Bonifacio, L. De Salvo, P. Pierini, N. Piovella, and C. Pellegrini, Spectrum, Temporal Structure, and Fluctuations in a High-Gain Free-Electron Laser Starting from Noise, Phys. Rev. Lett. 73, 70 (1994).

[17] R. Bonifacio, F. Casagrande, G. Cerchioni, L. de Salvo Souza, P. Pierini, and N. Piovella, Physics of the highgain FEL and superradiance, Riv. Nuovo Cimento 13, 1 (1990).

[18] L. Cveticanin, Analysis Techniques for the Various Forms of the Duffing Equation (John Wiley \& Sons, Ltd., New York, 2011), Chap. 4, pp. 81-137.

[19] M. Abramowitz and I. A. Stegun, Handbook of Mathematical Functions (Dover, New York, 1972).

[20] The lower sign in (27) can also be used to directly give the second expression for the field in (35).

[21] J. Barré, T. Dauxois, G. De Ninno, D. Fanelli, and S. Ruffo, Statistical theory of high-gain free-electron laser saturation, Phys. Rev. E 69, 045501 (2004).

[22] X. J. Wang, T. Watanabe, Y. Shen, R. K. Li, J. B. Murphy, T. Tsang, and H. P. Freund, Efficiency enhancement using electron energy detuning in a laser seeded free electron laser amplifier, Appl. Phys. Lett. 91, 181115 (2007).

[23] E. L. Saldin, E. A. Schneidmiller, and M. V. Yurkov, Statistical properties of radiation from VUV and X-ray free electron laser, Opt. Commun. 148, 383 (1998). 